moving group, of known age - between 50 million and 120 million years.

The authors identified the object using data from the Canada-France Brown Dwarf Survey InfraRed, and suggest that it has a planet-like mass four to seven times that of Jupiter.

Astron. Astrophys. 548, A26 (2012)

\section{Injectable gels spring into shape}

Sponge-like polymer gels 'remember' and regain their shape after being injected into mice, demonstrating their potential to deliver drugs and cells for tissue repair and regeneration.

A team led by David Mooney at Harvard University in Cambridge, Massachusetts, made the hydrogels - porous structures that absorb water - out of alginate, a complex sugar extracted from seaweed. The researchers placed starand heart-shaped gels in a syringe and injected them beneath the skin of mice; two days later, the materials had regained their original shapes. Gels that were preloaded with either growth-promoting proteins or cells and similarly injected into mice slowly released their contents before dissolving. Gel-delivered cells showed higher levels of survival and engraftment for up to 15 days than cells injected alone.

Proc. Natl Acad. Sci. USA

http://dx.doi.org/10.1073/

pnas.1211516109 (2012)

\section{MICROBIOLOGY}

\section{Ancient origin for gut microbiome}

Chimpanzees and humans share not only $96 \%$ of their DNA sequence, but also the basic make-up of their gut microbes.

The microbial communities that thrive in the human gut can typically be grouped into three 'enterotypes', each of which is dominated by a specific set of bacteria.

Howard Ochman at Yale

University in New Haven,

Connecticut, and his

colleagues categorized the enterotypes of 35 chimpanzees from Gombe Stream National Park in Tanzania. Between them, the chimpanzees also harboured three enterotypes, and these were similar in composition to those found in humans.

The results suggest that this variation in gut microbial groups is an ancient trait that preceded the split between humans and chimpanzees. Nature Commun. 3, 1179 (2012)

\section{NEURODEGENERATION}

\section{Parkinson's protein spreads}

Injecting the abnormal protein associated with Parkinson's disease into the brains of mice causes key symptoms of the disorder, seemingly by spreading from one neuron to another. The findings point to a causal link between the misfolded protein, $\alpha$-synuclein, and the neurodegeneration seen in Parkinson's.

Virginia Lee and her colleagues at the University of Pennsylvania in Philadelphia injected a synthetic abnormal form of $\alpha$-synuclein into the mouse striatum, a brain region to which the neurons that die in Parkinson's project. The injected protein clumped together to form Lewy bodies, a signature of Parkinson's disease. Neurons then began to die, and neurons that link up with those near the injection site also developed Lewy bodies, indicating cell-to-cell transmission of a-synuclein. Six months after the injection, the animals' motor coordination, strength and balance had deteriorated - all Parkinsonian symptoms.

Antibodies against a-synuclein could stop or slow down the spread of the disease, the authors suggest.

Science 338, 949-953 (2012)

For a longer story on this research, see go.nature.com/tcoflz

COMMUNITY CHOICE

The most viewed papers in science

\section{Faces warp as brain area is zapped}

Electrical stimulation of two specific brain regions distorts the perception of faces.

Facial recognition has been linked to the fusiform gyrus, but establishing a causal link and identifying the specific areas of this region involved have proved challenging. Josef Parvizi and his colleagues at Stanford University in California used three modes of brain-activity measurement to pinpoint a specific neural circuit involved in the visual perception of real faces. The authors stimulated electrodes implanted into the mid and posterior fusiform areas of a 45 -year-old man. He reported that the faces he saw in the room appeared so distorted that they almost looked like different people. He was still able to name famous faces and recognize other objects. Stimulating electrodes implanted into nearby brain areas had no effect.

Electrophysiology and functional magnetic resonance imaging data confirmed that these two fusiform areas were active when the volunteer was viewing faces.

J. Neurosci. 32, 14915-14920 (2012)

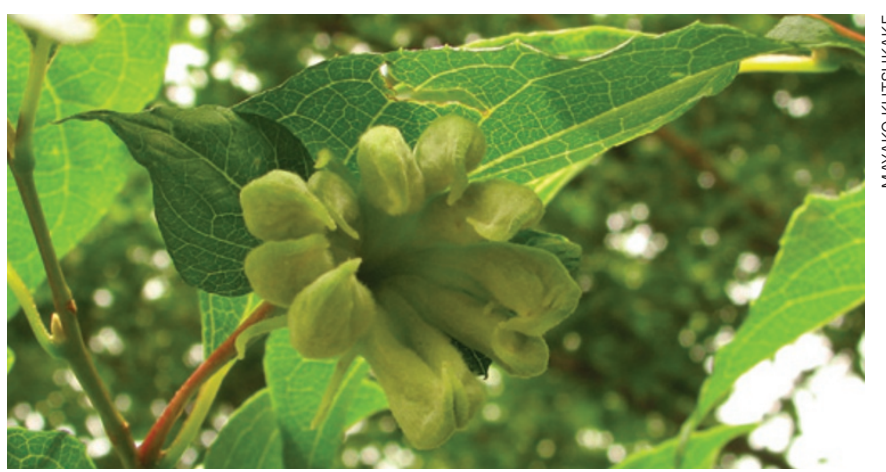

\section{ZOOLOGY}

\section{Aphids borrow plant plumbing}

Some aphid species build nests that are attached to plants and remain sealed for months so how do the insects avoid drowning in their own waste? It seems that they flush waste products using the fluidtransport systems of their host plants.

Takema Fukatsu of the National Institute of Advanced Industrial Science and Technology in Tsukuba, Japan, and his colleagues compared the nodule-like nests, or galls (pictured), of six species of aphid from the Hormaphidinae subfamily. By injecting various fluids, including some containing dye, into the closed galls, the team found that several species build sealed nests with spongy, absorbent walls that drain into the host plant's vascular system. By contrast, other species make open galls that have waxy, non-absorbent walls.

The authors suggest that aphid species evolved different strategies to balance colony hygiene with defence against predators and parasites. Nature Commun. 3, 1187 (2012)

\section{- NATURE.COM}

For the latest research published by Naturevisit:

www.nature.com/latestresearch 